\title{
Use of Virtual Rehabilitation to Improve the Symmetry of Body Temperature, Balance, and Functionality of Patients with Stroke Sequelae
}

\author{
Aristela de Freitas Zanona ${ }^{a-c, e}$ Raphael Fabricio de Souza ${ }^{a}$ d, f \\ Felipe J. Aidard,f,g Dihogo Gama de Matos ${ }^{f}$ Karine Morgana Batista Santos ${ }^{f}$ \\ Max da Conceição Paixão ${ }^{c}$ Priscila Yukari Sewo Sampaio ${ }^{c}$ \\ Heleno Almeida Junior d, g Katia Karina Monte-Silva ${ }^{a}$ b, e \\ a Postgraduate Program in Neuropsychiatry and Behavioral Sciences, Federal University of Pernambuco, Recife, \\ Brazil; ${ }^{b}$ Applied Neuroscience Laboratory, Federal University of Pernambuco - UFPE, Recife, Brazil; ' Department \\ of Occupational Therapy, Federal University of Sergipe - UFS, Lagarto, Sergipe, Brazil; ${ }^{\mathrm{d}}$ Department of Physical \\ Education, Federal University of Sergipe - UFS, São Cristovão, Sergipe, Brazil; e'Department of Physiotherapy, Federal \\ University of Pernambuco - UFPE, Recife, Brazil; ${ }^{f}$ Group of Studies and Research of Performance, Sport, Health and \\ Paralympic Sports - GEPEPS, Federal University of Sergipe - UFS, São Cristovão, Sergipe, Brazil; ${ }^{9}$ Graduate Program \\ in Master's Level in Physical Education, Federal University of Sergipe - UFS, São Cristovão, Sergipe, Brazil
}

\section{Keywords}

Body asymmetry $\cdot$ Stroke $\cdot$ Virtual reality .

Occupational therapy

\begin{abstract}
Background: Stroke rehabilitation that is based on the patients' needs, experiences, and priorities requires extensive knowledge and skills to capture and integrate the perspectives of the subject. Purpose: The objective of this study was to evaluate the acute effect of an occupational therapy protocol associated with virtual reality (VR) on the symmetry of body temperature (BTP), balance, and functionality of patients with stroke sequelae. Methods: Ten patients (69.84 \pm 7.55 years) diagnosed with stroke between 2 and 10 years earlier were evaluated during clinical care sessions integrated with VR games. Associated with games, all patients were stimulated to use both upper and lower limbs and distribute body weight symmetrically, and perceptual stimuli of bodyhalf training, alignment, postural control, and balance were
\end{abstract}

() 2018 S. Karger AG, Basel

E-Mail karger@karger.com www.karger.com/aon given. The variables of thermography (temperature $\left[{ }^{\circ} \mathrm{C}\right]$ and body asymmetry) of the upper and lower limbs, balance (Berg scale), and functionality were analyzed before and after the test. Results: BTP was reduced in the 4 upper and lower limb body regions of interest: the right arm ( $p=0.024$, Cohen's $d=1.02)$, previous direct hand ( $p=0.034$, Cohen's $d=1.22)$, right back hand ( $p=0.003$, Cohen's $d=1.85$ ) and Left ( $p=0.013$, Cohen's $d=0.92$ ), right thigh ( $p=0.035$, Cohen's $d=1.32$ ), and left thigh ( $p=0.047$, Cohen's $d=0.92$ ). The mean of the bilateral asymmetry variation of the arm in the anterior position at the pre test was classified according to the level of attention monitoring (which means that the asymmetry rate is above normal), changing its state at the end of the intervention to normal. There was an increase in the functional independence score $(p=0.015$, Cohen's $d=$ $0.50)$ and in the static and dynamic balance function ( $p=$ 0.001 , Cohen's $d=0.07$ ). Conclusion: VR associated with occupational therapeutic planning can amplify and potentiate neurological recovery following stroke.

(c) 2018 S. Karger AG, Basel

Prof. Aristela de Freitas Zanona

Department of Physiotherapy, Applied Neuroscience Laboratory, Postgraduate Program in Neuropsychiatry and Behavioral Sciences, Federal University of Pernambuco, Av. Prof. Moraes Rego, 1235 - Cidade Universitária, Recife - PE - CEP 50670-901 (Brazil) E-Mail aristela.zanona@ufs.br 


\section{Introduction}

Stroke is considered the leading cause of disability and death in the world [1], and those affected by stroke tend to have sequelae in the motor, sensory, cognitive, perceptual, emotional regulation, communication, and language functions [2].

In addition, motor dysfunctions and reduced sensitivity on one side of the body is known as hemiparesis [3]. Hemiparesis causes instability in maintaining control and postural alignment, as the patient tends to transfer body weight and hyperactivate the unaffected side while neglecting the affected side, making it hypoactive [4]. This condition causes the displacement of the center of gravity, resulting in imbalance and body asymmetry, which impairs the performance and execution of activities of daily living, predisposing the patient to the risk of falls and dependence on third parties $[2,5]$. These mismatches can be identified through asymmetry analysis, performed through thermography [6].

Therapeutic interventions aimed at this population aim to amplify and potentiate neurological recovery [3]. In this context, virtual reality (VR) has been used with the aim of stimulating pre-motor, perceptual, and cognitive abilities in order to maximize the performance of daily activities $[7,8]$. However, it is not yet clear in the literature whether a therapeutic program allied to VR can improve body asymmetry and balance. Moreover, it has not been ascertained whether the gains in these motor components are directly related to the decrease in dependence on caregivers. The general objective of the present study was to evaluate the acute effect of a VR protocol associated with conventional practices to improve the motor skills, and our specific objectives were to quantify (1) the body thermal symmetry (2) the balance, and (3) the functionality of patients with stroke sequelae.

\section{Methods}

Ten volunteers, 4 women (mean age $67.08 \pm 7.55$ years) and 6 men (mean age $72.6 \pm 7.2$ years), who had been diagnosed with stroke between 2 and 10 years earlier, were selected by convenience sampling. Of these, 8 patients were classified as having moderate disability (Grade 3 requires some help but is able to walk unassisted, can use a walking stick or walker), and 2 patients were classified as having mild deficiency (Grade 2 - unable to conduct all activities, but able to take care of one's own interests) according to the Post-Stroke Functional Scale - Modified Rankin Scale [9]. Patients who were diagnosed with stroke (according to the International Classification of Diseases), absence of hearing, or vision or speech problems did not present disorders resulting from degenerative diseases, fibromyalgia, chronic pain, or degenerative neuromuscular diseases and who were not undergoing any physical rehabilitation care during the collection period were included in the study.

We excluded from the study patients who, after being evaluated by the Fugl-Meyer scale, presented total absence of movement or sensation on one side of the body, mental illnesses, severe cognitive deficit (evaluated by the Mental State Examination Scale, cutoff point of 18 for illiterates and 20 for literate patients), disc herniation, and/or were not given a medical release for the practice of physical activity.

\section{Assessment of Body Symmetry}

In order to evaluate body symmetry, thermographic images were taken before and after clinical care according to the procedures recommended by the European Association of Thermology performed in a room properly prepared without natural light, with artificial lighting supplied by fluorescent lamps, without airflow (HM-01, São Paulo, Brazil), at a temperature between 23 and $24^{\circ} \mathrm{C}$, and relative humidity between 42 and $50 \%[10]$.

Individuals were instructed not to perform vigorous physical activity in the previous $24 \mathrm{~h}$, not to consume alcohol or caffeine, and not to use any type of cream or lotion on the skin in the last $6 \mathrm{~h}$ prior to the evaluation. Thermograms were obtained twice, before and shortly after the session. Each patient was advised to remain standing and not to make sudden movements, not to cross the arms, and not to scratch for a period of at least $10 \mathrm{~min}$ for acclimatization [11].

Images were obtained by using a C2 thermal imager (Flir System, Stockholm, Sweden). It has a measurement range of -10 to $150{ }^{\circ} \mathrm{C}, 2 \%$ accuracy, sensitivity $<0.10$, infrared spectral band of 7.5-14 $\mu \mathrm{m}$, refresh rate of $9 \mathrm{~Hz}$, and a resolution of $80 \times 60$ pixels at a distance of $1.5 \mathrm{~m}$, with emissivity set at 0.98 [12]. The images were then transferred to a computer and analyzed using Flir Tools software (Flir Systems, Stockholm, Sweden).

The selection of the body regions of interest (BRI) was based on the same rules used in studies by Marins et al. [11]. The anatomical points were as follows: (a) hand: junction of the 3rd proximal phalanx of the metacarpal with the 3rd styloid ulnar process; (b) forearm: cubital fossa up to distal forearm; (c) arm: cubital fossa up to axillary line; (d) thigh: $5 \mathrm{~cm}$ above the upper limit of the patella and inguinal line; (e) $5 \mathrm{~cm}$ below the patella and $10 \mathrm{~cm}$ above the malleolus. The corresponding anatomical points were also used in the posterior region.

After analysis, the body temperature delta $(\Delta \mathrm{TP})$ was calculated by subtracting the pre- and post-intervention body temperatures (BTP) and between the 2 limbs, considering asymmetry attention levels [11] (Table 1).

\section{Balance Scale}

The Berg balance scale (BBS) was used for static and dynamic assessment of balance in 14 tasks performed, such as posture changes from sitting to standing, standing tasks with determined duration, changes in support base, functional range, and change in the center of gravity in relation to the median line and support base. The scores on this scale range from 0 to 4 , totaling 56 points, where the higher the score, the better the balance and the lower the probability of falls [13]. 
Table 1. Asymmetry criteria

\begin{tabular}{ll}
\hline Level of attention & Asymmetries \\
\hline Normal & $\leq 0.4^{\circ} \mathrm{C}$ \\
\hline Monitoring & $\geq 0.5^{\circ} \mathrm{C}$, where it is advisable to reassess and verify if there is influence of some external factor \\
\hline Prevention & $\begin{array}{l}\text { Values between } 0.8 \text { and } 1.0^{\circ} \mathrm{C} \text {, where it is recommended a reduction in the load or even a suspension of the } \\
\text { training, and medical and/or physiotherapeutic evaluation }\end{array}$ \\
\hline Alarm & Values between 1.1 and $1.5^{\circ} \mathrm{C}$, immediate suspension of training and/or medical or physiotherapeutic evaluation \\
\hline Severity & $\begin{array}{l}\geq 1.6^{\circ} \mathrm{C} \text {, thus suggesting an asymmetry with a pathological characteristic or an important lesion with the } \\
\text { recommendation of medical and/or physiotherapeutic evaluation. }\end{array}$ \\
\hline
\end{tabular}

Functional Independence Measure (FIM)

The subjects' level of functional independence in their daily activities was assessed through the following aspects: personal care, sphincter control, mobility, locomotion, communication, and social knowledge. The level of functional independence is scored between 1 and 7 , where the need for care from third parties is measured according to the percentage of assistance that the patient receives in the 18 task units. After completion, the patient is classified in complete dependence (18-45 points), moderate independence (46-99), and complete independence (100-126) [14].

\section{Virtual Rehabilitation}

Evaluations and re-evaluations were performed with the validated and standardized instruments already mentioned. A total of 30 sessions were held each week, of $60 \mathrm{~min}$ each, in which VR games were selected to favor bilateral and symmetrical movements.

The equipment used was the Nintendo Wii (Nintendo ${ }^{\circledR}$ ) device with controls that were attached to the patient's wrists, a Kinect device to capture the patient's image and a projector that allowed the avatar to be viewed. The games used belong to Nintendo Sports ${ }^{\circledR}$. The VR protocol was drawn up using evidence from the literature, and after analysis, we selected boxing, bowling, snowboarding, swimming, and tennis games (alternating body side in each play) [15]. The games were selected from the skills that were required to achieve success in the match. In this way, the games require the patient to transfer weight to both sides of the body, increase the notion and perception of the middle of the body, and place the subject in situations where strategies to maintain balance are needed. Before each match, the patient analyzed each skill and was encouraged to think about the resolutions of problems that could occur during the game, such as loss of balance. In each session, lasting $60 \mathrm{~min}$, the games were alternated and each patient performed 2 different modalities of the sports mentioned above. Each game was repeated about 3 to 4 times until the patient reached the necessary skill for marking points in the match.

Parallel to the execution of the games, principles of the Bobath Concept were incorporated during the sessions. All patients were stimulated to use both upper and lower limbs and distribute body weight symmetrically, and perceptual stimuli of body-half training, alignment, postural control, and balance were given. Extra materials such as mirrors, parallel strips on the wall were used to aid in body alignment, as well as balance equipment such as boards or $85 \mathrm{~cm}$ balls, in which the patient was positioned standing or in the kneeling position, respectively, in order to promote the reach of maintaining a good base of support and alignment of the line and center of gravity

\section{Statistics}

To verify the distribution of the sample, the Shapiro-Wilk and Levene test for normality and homogeneity, respectively, were employed, and the paired $t$-test was performed. To verify the effect size, Cohen's d test was used. Results were considered statistically significant at $p<0.05$. All analyses were carried out using the Statistical Package for the Social Sciences (SPSS, IBM Inc., Chicago, IL, USA), version 20.0.

\section{Results}

The sample was predominantly male patients, mean age $65.66 \pm 9.43$, married, with incomplete elementary school, ischemic type of stroke, and a history of more than 1 stroke episode. All data are shown in Table 2.

When the pre-and post-intervention effects were evaluated, upper-limb BTP was reduced after the intervention in the BRI: the right arm $(p=0.024$, Cohen's $\mathrm{d}=$ $1.02)$, previous direct hand ( $p=0.034$, Cohen's $d=1.22)$, right back hand ( $p=0.003$, Cohen's $\mathrm{d}=1.85)$ and left $p=$ 0.013 , Cohen's $d=0.92)$. The lower limbs presented a significant reduction in BTP after the intervention in the following BRI: right posterior thigh $(p=0.035$, Cohen's $\mathrm{d}=1.32)$, and left thigh $(p=0.047$, Cohen's $\mathrm{d}=$ 0.92).

Table 3 shows in detail the BTP by body region verified before and after the intervention. The $\triangle \mathrm{TP}$ of the arm in the anterior position at the pre-moment was classified as the level of attention "monitoring," changing its state at the end of the intervention to "normal." Lower-limb evaluation revealed that the asymmetry of the anterior leg 
presented the level of attention "alarming" $\left(\Delta \mathrm{TP}^{\circ} \mathrm{C}\right.$ between 1.1 and $1.5^{\circ} \mathrm{C}$ ) in both pre-and post-intervention conditions (Table 4). Figure 1 shows the pre- and postintervention photochromatic variation by BRI quadrant analysis.

Regarding the variables functionality and balance, there was an increase in the FIM post-intervention score and the static and dynamic equilibrium function evaluated by the BBS. All values are shown in Table 5 .

\section{Discussion}

The objective of the present study was to evaluate the acute effect of the VR protocol associated with conventional practices working from the hypothesis that VR integrated into clinical care helps improve body symmetry, balance, and functionality in patients with stroke sequelae.

The use of technological resources to analyze body asymmetry was relevant because it is a reliable indicator of the effects of the intervention. A recent study [16] that used thermography in stroke patients (acute and subacute stage) indicated the use of this feature to monitor the function of both body sides in order to target the therapies of the rehabilitation team to asymmetry.

In this study, BTP was reduced in 4 BRI of the upper limbs and 2 BRI of the lower limbs. There was a significant reduction in temperature on the right side of the body, in the arm $(p=0.024)$, and in the anterior $(p=$ $0.034)$ and posterior $(p=0.003)$ hand identified as the most active body portion even in bilateral activity. In the lower limbs, a reduction in BTP was observed in both right $(p=0.035)$ and left $(p=0.047)$ portions but only in the thighs of the volunteers - a region that was closer to the hip - which performed important work in the stabilization of body movements.

Thermography studies the BTP of the epidermis and can be modified according to muscle activity. Thus, the reduction of BTP can be identified as a beneficial factor. As with the increase of motor work during VR games, there was an increase in the muscular activity interfering in thermoregulation, thereby increasing the production of sweat and cooling of the skin. Therefore, a reduction of the BTP of the epidermis can be interpreted as muscular activation, which occurs similarly in other physical exercises and with consequent relevance to the patient with stroke for developing a dynamic action.
Table 2. Characteristics of the sample

\begin{tabular}{|c|c|c|}
\hline Variable & Patients & $\%$ \\
\hline \multicolumn{3}{|l|}{ Gender } \\
\hline Men & 6 & 60.0 \\
\hline Women & 4 & 40.0 \\
\hline \multicolumn{3}{|l|}{ Age, years, mean $\pm \mathrm{SD}$} \\
\hline Men & $63.16 \pm 10.60$ & \\
\hline Women & $71 \pm 4.58$ & \\
\hline \multicolumn{3}{|l|}{ Marital status } \\
\hline Not married & 0 & 0.00 \\
\hline Married & 6 & 60.0 \\
\hline Divorced & 0 & 0.00 \\
\hline Widower & 4 & 40.0 \\
\hline \multicolumn{3}{|l|}{ Education } \\
\hline Illiterate & 3 & 22.25 \\
\hline Elementary school incomplete & 4 & 44.4 \\
\hline Complete primary education & 1 & 11.1 \\
\hline Incomplete high school & 0 & 00.0 \\
\hline Complete high school & 2 & 22.25 \\
\hline \multicolumn{3}{|l|}{ Type of stroke } \\
\hline Ischemic & 7 & 70.0 \\
\hline Hemorrhagic & 4 & 40.0 \\
\hline \multicolumn{3}{|l|}{ Number of stroke episodes } \\
\hline 1 & 3 & 30.0 \\
\hline 2 & 7 & 70.0 \\
\hline \multicolumn{3}{|l|}{ Dimidio affected } \\
\hline Left & 5 & 50.0 \\
\hline Right & 5 & 50.0 \\
\hline
\end{tabular}

After the intervention, the asymmetry of the anterior (right and left) arm changed from the level requiring monitoring attention $\left(\Delta \mathrm{TP} 0.5^{\circ} \mathrm{C}\right)$ to normal $(\Delta \mathrm{TP}$ $0.3^{\circ} \mathrm{C}$ ) [11]. Thus, it was hypothesized that VR presented substantial evidence of improvement, especially in upper limb indicating that VR allows the modulation of arm and hand functions through forced use during play.

In the BRI of the lower limbs, the attention level was continuously "alarming." This fact can be explained by the patient's difficulty in effectively coordinating all the demands of the activity with the required skills. Since the games required the focus to be on the upper limbs, it may have occurred that the patients paid selective attention to the upper limbs despite the therapist's constant commands to transfer weight equally to the lower limbs.

Thermal imaging has been widely used in medical practice for diagnostic purposes and monitoring of various physical disorders [17]. Several studies have used thermography in diagnostic cases of neoplasias [18], muscle damage $[19,20]$, ischemia in diabetes [21], and 
Table 3. Means of BTP $\left({ }^{\circ} \mathrm{C}\right)$ pre and post BRI

\begin{tabular}{lllll}
\hline BRI & BTP $\left({ }^{\circ} \mathrm{C}\right)$ pre test & BTP $\left({ }^{\circ} \mathrm{C}\right)$ post test & $p$ value & Cohen's d \\
\hline Upper limbs & & & & \\
$\quad$ Right forearm & $32.26 \pm 0.99$ & $31.03 \pm 1.37^{*}$ & 0.024 & 1.02 \\
Left forearm & $32.01 \pm 0.89$ & $31.34 \pm 1.56$ & 0.108 & 0.52 \\
Right rear arm & $31.07 \pm 1.13$ & $30.77 \pm 1.46$ & 0.511 & 0.22 \\
Left rear arm & $31.22 \pm 1.03$ & $30.67 \pm 1.51$ & 0.339 & 0.42 \\
Forearm anterior straight & $31.66 \pm 1.18$ & $31.01 \pm 1.67$ & 0.420 & 0.44 \\
Left forearm & $31.82 \pm 0.81$ & $31.13 \pm 1.26$ & 0.144 & 0.65 \\
Right forearm & $31.63 \pm 1.02$ & $31.33 \pm 1.43$ & 0.393 & 0.24 \\
Left forearm & $31.61 \pm 1.45$ & $31.01 \pm 1.59$ & 0.260 & 0.39 \\
Previous direct hand & $31.93 \pm 0.96$ & $30.37 \pm 1.52^{*}$ & 0.034 & 1.22 \\
Previous left hand & $31.85 \pm 1.18$ & $30.97 \pm 1.23$ & 0.239 & 0.73 \\
Rear right hand & $30.86 \pm 0.59$ & $29.58 \pm 0.78^{*}$ & 0.003 & 1.85 \\
Left back hand & $30.65 \pm 1.05$ & $29.75 \pm 0.88^{*}$ & 0.013 & 0.92 \\
Lower members & & & & 0.78 \\
Anterior thigh right & $31.01 \pm 1.00$ & $30.33 \pm 0.71$ & 0.086 & 0.78 \\
Anterior thigh left & $30.95 \pm 1.20$ & $30.26 \pm 0.88$ & 0.081 & 0.66 \\
Right posterior thigh & $31.62 \pm 0.63$ & $30.78 \pm 0.64^{*}$ & 0.035 & 1.32 \\
Left posterior thigh & $31.88 \pm 0.98$ & $30.93 \pm 1.08^{*}$ & 0.047 & 0.92 \\
Right leg & $31.10 \pm 1.54$ & $30.28 \pm 1.39$ & 0.314 & 0.10 \\
Forelegs left & $31.41 \pm 1.72$ & $30.32 \pm 1.31$ & 0.097 & 0.71 \\
Right rear leg & $30.28 \pm 0.78$ & $29.26 \pm 1.11$ & 0.087 & 0.91 \\
Left back leg & $30.26 \pm 1.39$ & $29.42 \pm 0.77$ & 0.920 & 0.31 \\
\hline
\end{tabular}

$* p<0.05$.

BTP, body temperature; BRI, body region of interest.

Table 4. Médias da variação de temperatura bilateral $-\Delta \mathrm{TP}\left({ }^{\circ} \mathrm{C}\right)$

\begin{tabular}{lllll}
\hline BRI & Before $\Delta \mathrm{TP}\left({ }^{\circ} \mathrm{C}\right)$ & Nível de atenção & After $\Delta \mathrm{TP}\left({ }^{\circ} \mathrm{C}\right)$ & Nível de atenção \\
\hline Upper limbs & & & \\
$\quad$ Anterior arm & $0.5 \pm 0.4$ & To monitor & $0.3 \pm 0.3$ & Normal \\
$\quad$ Rear arm & $0.5 \pm 0.2$ & To monitor & $0.5 \pm 0.3$ & To monitor \\
Forearm & $0.7 \pm 0.3$ & To monitor & $0.6 \pm 0.4$ & To monitor \\
$\quad$ Posterior forearm & $0.6 \pm 0.4$ & To monitor & $0.5 \pm 0.5$ & To monitor \\
$\quad \begin{array}{l}\text { Previous hand } \\
\text { Rear hand }\end{array}$ & $0.4 \pm 0.3$ & To monitor & $0.7 \pm 0.8$ & To monitor \\
Lower Members & $0.5 \pm 0.2$ & To monitor & $0.5 \pm 0.4$ & To monitor \\
$\quad$ Anterior thigh & $0.4 \pm 0.3$ & & & To monitor \\
$\quad$ Posterior thigh & $0.6 \pm 0.4$ & To monitor & $0.4 \pm 0.4$ & To monitor \\
Anterior leg & $1.2 \pm 0.9$ & To monitor & $0.4 \pm 0.4$ & Alarm \\
Posterior leg & $0.6 \pm 0.5$ & Alarm & $1.2 \pm 0.8$ & To monitor \\
\hline
\end{tabular}

BRI, body region of interest below $0.4^{\circ} \mathrm{C}$ - normal; $0.5-0.7^{\circ} \mathrm{C}$ - requires monitoring; $0.8-1.0^{\circ} \mathrm{C}-$ requires prevention; $1.1-1.5^{\circ} \mathrm{C}$ - gravity alarm; $>1.6^{\circ} \mathrm{C}$ - high severity (Marins et al. [11]).

evaluation and recovery of muscle function [17]. However, few studies have used this tool in the field of neurology and future research is suggested.

Following analysis of the balance results, it was verified that VR can be an opportunity to retrain postural control essential in the composition of the therapeutic planning of rehabilitation. Considered a technique based on biofeedback, it has presented positive evidence for the improvement of motor control capacity allowing a better perception of body movements. 

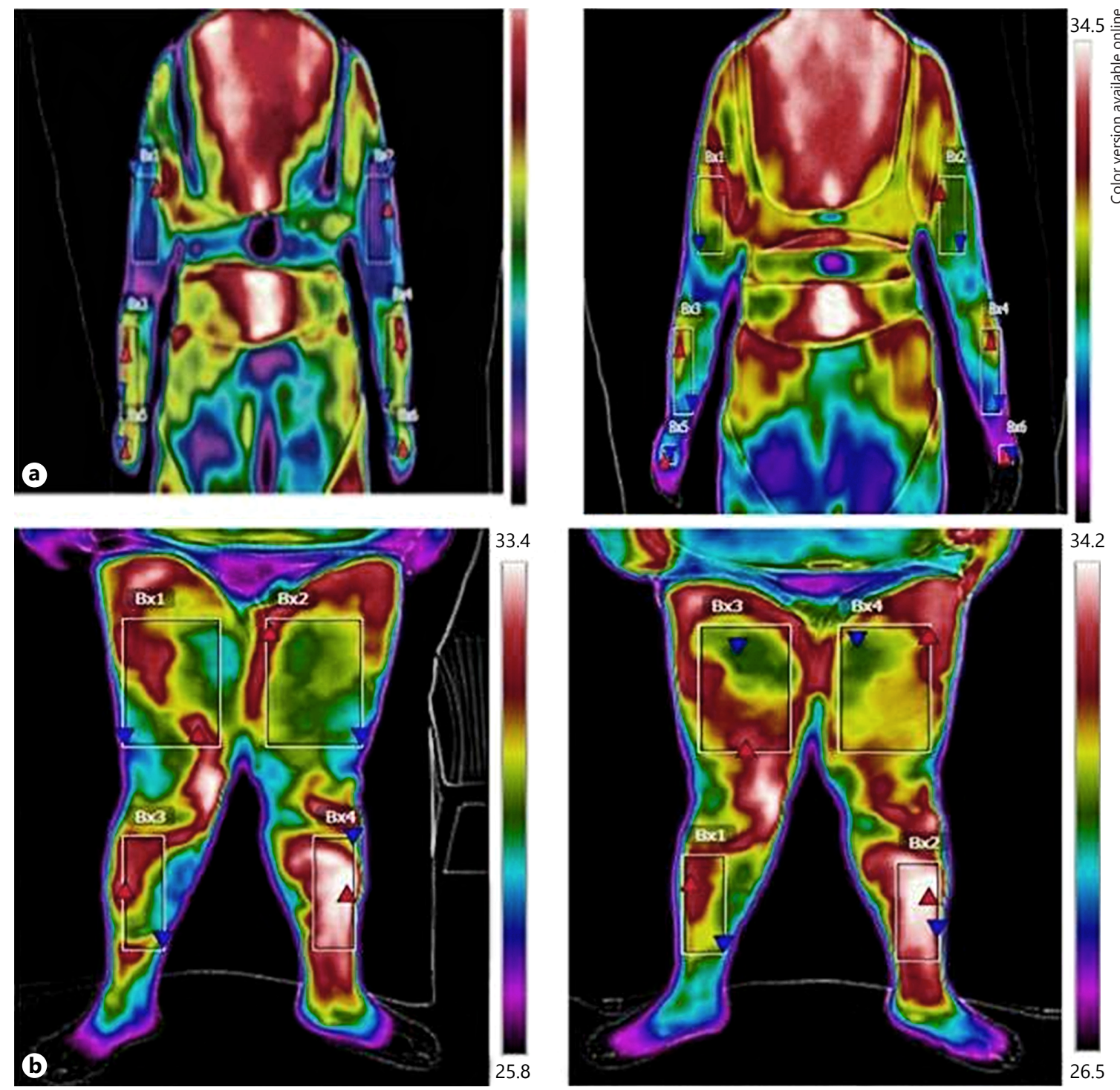

Fig. 1. Thermograms of the upper and lower limbs pre and post intervention (anatomical position). a Thermographic analysis posterior view of body segments: arm, forearm and hand. b Thermographic analysis anterior view of body segments: thigh and leg source. Personal archive.

The greater the significant increase in the Berg balance scale score, the lower the likelihood of falls and the greater the degree of body control of the subject [13]. According to Bassett et al. [22], in the range of 56-54 points, each lesser point is associated with a $3-4 \%$ increase in the risk of falls. From 54 to 46 , a change in 1 point is associated with a $6-8 \%$ increase in the odds and below 36 points, the risk of falls is almost $100 \%$. After the interventions, scores increased by an average of 6.5 points, which is indicative of a significant improvement in body balance and a decrease in the risk of falls.
Table 5. Results of functional independence and balance

\begin{tabular}{lclll}
\hline Instrument & Pre test & Post test & $p$ value & Cohen's d \\
\hline FIM & $58.75 \pm 31.36$ & $73.0 \pm 25.17$ & $0.015^{*}$ & 0.50 \\
BBS & $37.5 \pm 9.81$ & $44.0 \pm 8.66$ & $0.001^{*}$ & 0.07 \\
\hline
\end{tabular}

$* p<0.05$.

Values were calculated as mean and SD.

FIM, functional independence measure; BBS, Berg balance scale. 
For functional independence, there was a significant difference in FIM pre- and post-intervention scores, thus suggesting that the use of VR can be effective in regaining function after stroke. This result indicates that although the exercises were not directly executed to favor self-care, this improvement may be associated with maximization of balance and body symmetry [23].

According to previous studies [7], there was a positive association between training with VR and improvement in the performance of basic and instrumental activities of daily living as well as a significant reduction in everyday tasks. This may be explained by the large number of repetitions and specific movements that are associated with continuous biofeedback, contributing to motor learning.

It is possible to infer that the improvement in functionality is directly related to the improvement in the learning retention process observed throughout the intervention protocol, particularly stimulated by the learning transfer phase followed by exposure to VR.

The application of VR allied to occupational therapy significantly improved balance and functionality albeit with a small improvement in body symmetry. The application of VR in the therapeutic sessions was identified as an adequate resource adapted to individualized patient needs. However, it is recommended that this be used as an auxiliary resource and not as a substitute for the conventional therapeutic session. In addition, VR provides the patient with greater engagement motivation and involvement in therapy.

Our study had some limitations. The sample number was limited to 10 participants. Originally, we started evaluations with a larger number of participants. However, they had difficulties arriving at the place of attendance for the sessions, and the research took a long time to be completed. Behavioral assessments of balance and functionality, although widely used in clinical practice, may present subjectivity in application and interpretation. Because of that, we suggest the use of equipment such as the balancing platform and applications of complementary scales of functionality, based on the International Classification of Functioning, Disability and Health.

\section{Future Research Lines}

The use of technologies to assess and treat conditions of disability has been widely disseminated among health professionals. In the practice of rehabilitation, uniting knowledge of related areas such as engineering can be useful not only to reliably identify problems in bodily functions but also to prove the efficacy of therapeutic techniques since, until then, they were considered empirical. The thermography (used in the evaluation) and the VR (in the treatment) maximized the conventional therapeutic action and offered the professional and the patient modernity of easy access.

\section{Acknowledgments}

None.

\section{Ethical Approval}

The study protocol was approved by the Ethics Committee of the University Hospital of the Federal University of Sergipe (CAAE: 64009616.5.0000.5546), Aracaju, Brazil. The study was conducted in accordance with the ethical standards laid down in the 1964 Declaration of Helsinki.

\section{Disclosure Statement}

Authors declare they have no competing financial interests to disclose.

\section{Funding Sources}

The authors have no funding to declare.

\section{Author Contribution}

A.F.Z. and K.K.M.-S. conceptualized the paper, P.Y.S.S., D.G.M., K.M.B.S., and M.C.P. drafted the paper, H.A.J. manipulated the thermographic camera, F.J.A. performed the statistical analysis, and R.F.S. edited it.

References A, Jumaa M, Schroeder JL: Streptococcus mutans: a potential risk factor in recurrent hemorrhagic stroke. Cureus 2017;9:e1264.

-2 Cazzoli D, MürI RM, Schumacher R, Von ARX S, Chaves S, Gutbrod K, Bohlhalter S, Bauer D, Vanbellingen T, Bertschi M, Kipfer S, Rosenthal CR, Kennard C, Bassetti CL, Nyffeler T: Theta burst stimulation reduces disability during the activities of daily living in spatial neglect. Brain 2012;135(pt 11): 3426-3439.

3 Teasell R, Hussein N: Brain reorganization, recovery and organized care. Stroke Rehabil Clin Hand 2016:1-34. 
4 montastruc J, AmarantinI D, Lambert V: Main determinants of weight-bearing asymmetry in hemiplegic stroke patients. Ann Phys Rehabil Med 2016;59:59-69.

$\checkmark 5$ Kim YK, Jung JH, Shin SH: A comparison of the effects of repetitive transcranial magnetic stimulation (rTMS) by number of stimulation sessions on hemispatial neglect in chronic stroke patients. Exp Brain Res 2015;23:283-289.

6 Neves EB, Vilaça-Alves J, Rosa C, Reis VM: Thermography in neurologic practice. Open Neurol J 2015;9:24-27.

7 Saposnik G, Mamdani M, Bayley M, Thorpe KE, Hall J, Cohen LG, et al: Effectiveness of virtual reality exercises in stroke rehabilitation (EVREST): rationale, design, and protocol of a pilot randomized clinical trial assessing the Wii gaming system. Int J Stroke 2010; 5:47-51.

8 Schiavinato AM, Machado BC, Pires MA: Influência da realidade virtual no equilíbrio com portador de lesão cerebelar-estudo de caso. Rev Neurocienc 2011;19:119-127.

-9 Wilson JTL, Harendran A, Grant M: Improving the assessment of outcomes in stroke: use off a structured interview to assign grades on the modified rankin scale. Stroke 2002;33: 2243-2246.
10 Fernández-Cuevas I, Sillero-Quintana M, Garcia-Concepcion MA, et al: Monitoring skin thermal response to training with infrared thermography. New Stud Athlet 2014;29:57-71.

11 Marins JC, Moreira DG, Cano SP, Quintana MS, Soares DD, Fernandes AA, Silva FS, Costa CM, Amorim PR: Time required to stabilize thermographic images at rest. Infrared Phys Technol 2014;65:30-35.

12 Steketee J: Spectral emissivity of skin and pericardium. Phys Med Biol 1973;18:686-694.

113 Berg KO, Wood-Dauphine SL, Gayton D: Measuring balance in the elderly: preliminary development of an instrument. Physiother Can 1989;41:304-311.

14 Nagai K, Yamaguchi F: Improved functional Independence measure facilitates return to home after paralyzed upper-limb training: a case report. J Phys Ther Sci 2017;29:954-958.

15 Yates M, Kelemen A, Sik Lanyi C: Virtual reality gaming in the rehabilitation of the upper extremities post-stroke. Brain Inj 2016;30: 855-863.

16 Karaszewski B, Carpenter TK, Thomas RG, et al: Relationships between brain and body temperature, clinical and imaging outcomes after ischemic stroke. J Cereb Blood Flow Metab 2013;33:1083-1089.
17 Neves EB, Vilaça-Alves J, Moreira TR, Lemos RJ, Reis VM: The thermal response of biceps brachii to strength training. Gaz Medic Italiana Arch per le Scie Medic 2016;175:391-399.

18 Suganthi SS, Ramakrishnan S: Analysis of breast thermograms using gabor wavelet anisotropy index. J Med Sys 2014;38:1-7.

19 Bandeira F, Moura MAM, Souza MA, Nohama P, Neves EB: Pode a termografia auxiliar no diagnóstico de lesões musculares em atletas de futebol? Rev Bras Med Esporte 2012;18:246-251.

20 Bandeira F, Neves EB, Moura MAM, Nohama P: A termografia no apoio ao diagnóstico de lesão muscular no esporte. Rev Bras Med Esporte 2014;20:59-64.

21 Van Netten JJ, Van Baal JG, Liu C, et al: Infrared thermal imaging for automated detection of diabetic foot complications. J Diabetes Sci Technol 2013;7:1122-1129.

22 Bassett AM, Siu KC, Honaker JA: Functional measures for fall risk in the acute care setting: a review. West J Nurs Res 2017, Epub ahead of print.

23 Silva WHS, Lopes GLB, Yano KM, et al: Effect of a rehabilitation program using virtual reality for balance and functionality of chronic stroke patients. Motriz Rev Educ Fis 2015;21:33-40. 